\title{
The oncolytic Newcastle disease virus as an effective immunotherapeutic strategy against glioblastoma
}

\author{
Joshua A. Cuoco, DO, MS, ${ }^{1-3}$ Cara M. Rogers, DO, ${ }^{1-3}$ and Sandeep Mittal, MD, FRCSC ${ }^{1-4}$ \\ ${ }^{1}$ Carilion Clinic Neurosurgery, Roanoke; ${ }^{2}$ Fralin Biomedical Research Institute at Virginia Tech Carilion School of Medicine, \\ Roanoke; ${ }^{3}$ School of Neuroscience, Virginia Tech, Blacksburg; and ${ }^{4}$ Department of Biomedical Engineering and Mechanics,
} Virginia Tech, Blacksburg, Virginia

\begin{abstract}
Glioblastoma is the most frequent primary brain tumor in adults, with a dismal prognosis despite aggressive resection, chemotherapeutics, and radiotherapy. Although understanding of the molecular pathogenesis of glioblastoma has progressed in recent years, therapeutic options have failed to significantly change overall survival or progression-free survival. Thus, researchers have begun to explore immunomodulation as a potential strategy to improve clinical outcomes. The application of oncolytic virotherapy as a novel biological to target pathogenic signaling in glioblastoma has brought new hope to the field of neuro-oncology. This class of immunotherapeutics combines selective cancer cell lysis prompted by virus induction while promoting a strong inflammatory antitumor response, thereby acting as an effective in situ tumor vaccine. Several investigators have reported the efficacy of experimental oncolytic viruses as demonstrated by improved long-term survival in cancer patients with advanced disease. Newcastle disease virus (NDV) is one of the most well-researched oncolytic viruses known to affect a multitude of human cancers, including glioblastoma. Preclinical in vitro and in vivo studies as well as human clinical trials have demonstrated that NDV exhibits oncolytic activity against glioblastoma, providing a promising avenue of potential treatment. Herein, the authors provide a detailed discussion on NDV as a mode of therapy for glioblastoma. They discuss the potential therapeutic pathways associated with NDV as demonstrated by in vitro and in vivo experiments as well as results from human trials. Moreover, they discuss current challenges, potential solutions, and future perspectives in utilizing NDV in the treatment of glioblastoma.
\end{abstract}

https://thejns.org/doi/abs/10.3171/2020.11.FOCUS20842

KEYWORDS glioblastoma; central nervous system tumors; neuro-oncology; immune-based therapy; experimental oncolytic virotherapy; vaccine-based immunotherapeutics

A MONG primary malignant intracranial tumors, glioblastoma (GBM) is the most common and carries a dismal prognosis. Despite standard-of-care treatment consisting of maximal safe resection followed by radiation therapy and temozolomide chemotherapy, the median overall survival (OS) and progression-free survival (PFS) for GBM remain 14.6 and 7 months, respectively. ${ }^{1,2}$ Numerous factors contribute to its pathogenesis on the macroscopic level, including the blood-brain barrier, diffuse infiltration, and locations within nonresectable eloquent cortex. Moreover, on the molecular level, GBM drives pathogenicity with intratumoral heterogeneity, glioma stem cells, and a multitude of mechanisms by which tumor cells can induce local as well as widespread systemic immunosuppression., ${ }^{2,3}$ Given the poor prognosis with current treatment options, vaccine-based immunotherapeutics have emerged as a promising modality to circumvent GBM pathogenesis and ultimately improve clinical outcomes.

The application of oncolytic virotherapy as a novel biological to target pathogenic signaling in GBM has brought new hope to the field of neuro-oncology. ${ }^{2}$ This class of immunotherapeutics combines selective cancer cell lysis prompted by virus induction while promoting a strong inflammatory antitumor response, thereby acting as an effective in situ tumor vaccine. ${ }^{4}$ Several investigators have reported the efficacy of experimental oncolytic viruses as demonstrated by improved long-term survival in cancer patients with advanced disease. ${ }^{2,5-9}$ Indeed, several viruses have been studied to target GBM, including herpes simplex virus, vaccinia virus, and adenovirus. ${ }^{2,6,7,10}$ However, Newcastle disease virus (NDV) is one of the most wellstudied oncolytic viruses known to affect a multitude of human cancers..$^{8,9,11-13}$ Preclinical in vitro studies as well

ABBREVIATIONS ASI = active specific immunization; ATV-NDV = NDV-infected antitumor vaccination; $B I U=$ billion infectious units; DTH = delayed type hypersensitivity; $F$ = fusion protein; GBM = glioblastoma; HN = hemagglutinin-neuraminidase; IFN = interferon; NDV = Newcastle disease virus; OS = overall survival; PFS = progression-free survival.

SUBMITTED September 28, 2020. ACCEPTED November 16, 2020.

INCLUDE WHEN CITING DOI: 10.3171/2020.11.FOCUS20842. 


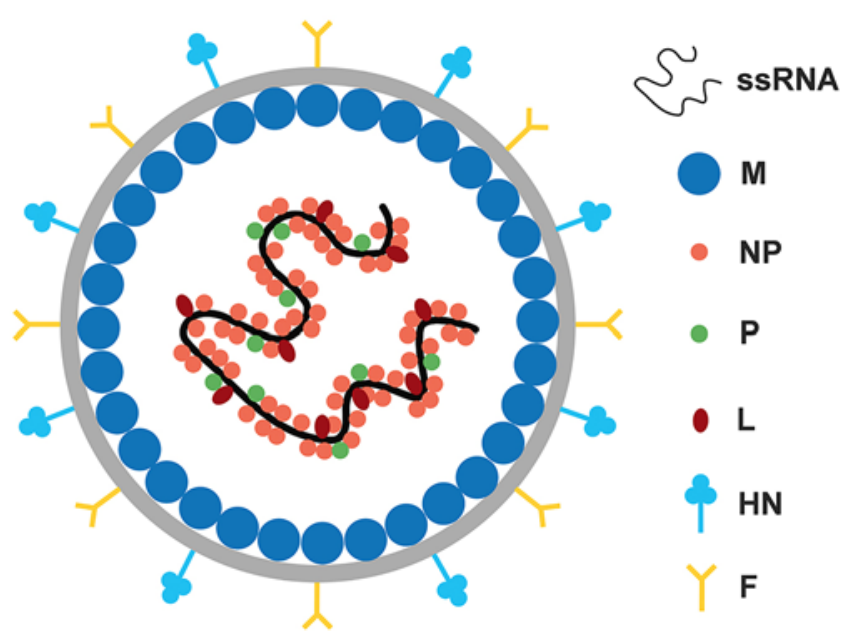

FIG. 1. Structure of NDV. The six main viral proteins include nucleoprotein $(N P)$, phosphoprotein $(P)$, matrix protein $(M)$, fusion protein $(F)$, hemagglutinin-neuraminidase $(\mathrm{HN})$, and polymerase protein (L). SsRNA = single-stranded RNA.

as clinical trials have shown that NDV exhibits oncolytic activity against GBM. ${ }^{12}$ Here, we provide a detailed discussion on NDV for the treatment of GBM. We discuss the potential therapeutic pathways associated with NDV as demonstrated by preclinical data as well as human trials. Moreover, we discuss current challenges, potential solutions, and future perspectives in utilizing NDV for GBM therapy.

\section{Virology of Newcastle Disease Virus}

NDV, also known as "avian paramyxovirus serotype 1" (APMV-1), was first identified in Java, Indonesia, in 1926 and in Newcastle-upon-Tyne, England, in $1927 .{ }^{14} \mathrm{It}$ is a nonsegmented, negative-sense, single-stranded RNA enveloped virus from the family Paramyxoviridae and genus Avulavirus. ${ }^{15}$ The genome encodes for six main viral proteins including nucleoprotein (NP), phosphoprotein $(\mathrm{P})$, matrix protein $(\mathrm{M})$, fusion protein $(\mathrm{F})$, hemagglutinin-neuraminidase $(\mathrm{HN})$, and polymerase protein (L; Fig. 1). ${ }^{15,16}$ Virus particles exhibit projections covering the surface encoated within a viral envelope. F and HN proteins expressed on the surface allow the virus envelope to fuse with host cellular membranes. ${ }^{15,16}$ Importantly, NDV exhibits preferential selectivity to replicate within tumor cells rather than normal cells..$^{17,18}$ Also, intracellular insertion of viral $\mathrm{HN}$ and $\mathrm{F}$ surface antigens results in a strong inflammatory response with the secretion of cytokines, chemokines, and type I interferons (IFNs). ${ }^{15,16,18,19}$ These proteins in turn modulate tumor cell surface markers and induce apoptosis downstream. ${ }^{19}$ Specifically, NDV can achieve oncolysis via activation of the extrinsic or intrinsic caspase-dependent pathways of cell death, activation of the endoplasmic eIF2a kinase PERK and caspase-12, or secretion of tumor necrosis factor $-\alpha$ from the infected tumor cells, among other mechanisms. ${ }^{19-21}$

Although NDV predominantly affects domestic poultry and otherwise poses no major threat to humans, rare infections resulting in conjunctivitis and mild flu-like symptoms have been reported. ${ }^{22,23}$ Nevertheless, in preclinical animal model studies this virus has been found to be an effective oncolytic agent against various cancers including neuroblastoma; fibrosarcoma; colorectal, hepatocellular, gastric, lung, breast, and prostate carcinoma; and, importantly, GBM. ${ }^{24}$ Indeed, the fact that NDV can replicate up to $10,000 \times$ more effectively in human cancer cells than in nonneoplastic cells prompted interest in utilizing it as an antineoplastic tool. ${ }^{17} \mathrm{~A}$ propensity to selectively replicate in cancerous cells translates into a higher safety and nonneurotropic profile than other oncolytic viruses. ${ }^{17-19}$ Based on virulence observed in birds, NDV can be subdivided into three groups: 1) lentogenic (low virulence), 2) mesogenic (intermediate virulence), and 3) velogenic (high virulence). ${ }^{24}$ Moreover, based on the composition of the F protein, NDV can be classified into either lytic or nonlytic strains. ${ }^{15,24}$ Several NDV strains (e.g., 73-T, MTH-68/H, HUJ, Ulster, LaSota, and AF2240) exhibit oncolytic activity in GBM, and the MTH$68 / \mathrm{H}, \mathrm{HUJ}$, and Ulster strains have been primarily evaluated in early phase studies in patients with GBM..$^{12,25-27}$

\section{Preclinical Data Apoptosis}

Programmed cell death is part of the cellular innate response to viral infections. The intentional sacrifice of infected cells serves as an attempt to prevent further spread of the virus to nearby cells. This concept applies to oncolytic virotherapy whereby viral infection of a cancer cell line may trigger apoptosis via one of several mechanisms. ${ }^{20}$ Washburn and Schirrmacher analyzed the immunomodulatory effects of infection of the NCH149 GBM cell line with NDV. ${ }^{19}$ They demonstrated that infection with a live virus or via cell surface adsorption of an ultraviolet-inactivated replication-deficient virus induced expression of proinflammatory IFN- $\alpha$ and IFN- $\beta$ as well as the chemokines RANTES (regulated upon activation, normal $\mathrm{T}$ cell expressed and presumably secreted, also known as "CCL5") and IFN- $\gamma$-inducible protein-10 (IP10). ${ }^{19}$ Moreover, viral infection upregulated human leukocyte antigens and cell adhesion molecules, thereby allowing improved tumor-associated antigen recognition and induction of apoptosis..$^{19}$ Fábián et al. evaluated NDV variant MTH-68/H cytotoxicity in the LNZTA3WT4 GBM cell line with inducible/repressible expression of $\mathrm{p} 53 .{ }^{21}$ They demonstrated no difference in MTH-68/H sensitivity in p53-expressing and p53-depleted states, which indicated that the induction of apoptosis was independent of p53 status. ${ }^{21}$ Rather, the oncolytic effect of MTH$68 / \mathrm{H}$ was determined to be attributable to the induction of endoplasmic reticulum stress (i.e., phosphorylation of protein kinase R-like endoplasmic reticulum kinase and eukaryotic initiation factor 2) with downstream activation of caspase-3 and caspase-12 leading to p53-independent apoptotic cell death. ${ }^{21}$

\section{Necroptosis}

Although apoptosis has traditionally been considered the mechanism of cellular death with viral therapy, some literature has suggested that necroptosis, or programmed 
necrosis, may also serve a role. ${ }^{28}$ Koks et al. investigated NDV Hitchner B1 strain in an orthotopic, syngeneic murine GL261 GBM model. ${ }^{29}$ They demonstrated that the survival of mice whose tumor was injected with the virus was significantly longer than that of controls. Contrary to the prior belief that immunosuppression must be present in order for oncolytic virotherapy to be effective, this study showed that the virus is capable of stimulating immunogenic cell death and necroptosis rather than apoptosis. ${ }^{29}$ Specifically, NDV treatment was observed to induce necrotic cell death without evidence of typical apoptotic stigmata. However, cell death did not occur in a chaotic, unorganized manner; rather, a progressive reduction in survival from 24 to 96 hours posttreatment was observed. Moreover, pretreatment with Necrostatin-1, a known in vitro synthetic inhibitor of receptor-interacting protein kinase 1-mediated necroptosis, rescued the virally induced cytopathic effect of NDV. This suggested that NDV induces glioma cell death via necroptosis in this model. Moreover, this was the first report indicating the induction of long-term, tumor-specific immunological memory after NDV oncolytic virotherapy in the GL261 model. ${ }^{29}$

\section{Type I Interferons}

Type I IFN induction serves an important role in tumor immunogenicity, including antigen presentation, dendritic cell maturation, activation of memory cytotoxic $\mathrm{T}$ lymphocytes, activation of natural killer cells, and recruitment of neutrophils. ${ }^{30,31}$ Loss of expression of the type I IFN gene cluster in cancer cells partly disables the ability of tumor cells to trigger tumor interactions with the adaptive immune response. Thus, cancer cells lacking this gene cluster would theoretically be more receptive to viral replication and display a more effective oncolytic response than cells with a competent type I IFN gene cluster. ${ }^{31}$ Importantly, a common characteristic in GBM is loss of CDKN2A (p16 $6^{\mathrm{INK} 4}$ ), located close to the cluster of type I IFN genes on chromosome 9p21. García-Romero and coworkers analyzed the CDKN2A-IFN1 gene cluster in 1018 gliomas, including GBM, and assessed the oncolytic effect of NDV in six GBM cancer stem cells ex vivo and in a murine model. ${ }^{31}$ They found that $>50 \%$ of GBM patients exhibited a type I IFN gene deletion. Moreover, they reported that GBM susceptibility to NDV was dependent on the loss of the type I IFN gene cluster. These data highlight that GBM patient stratification based on the $C D K N 2 A-I F N$ gene cluster deletion may serve as a sensitivity biomarker to determine patients who may respond to NDV oncolytic therapy. ${ }^{31}$

\section{Mesenchymal Stem Cells}

Mesenchymal stem cells have been reported to modulate tumor growth as well as exhibit a tumoricidal effect in experimental GBM.,32 Kazimirsky and colleagues examined the ability of these cells to deliver the virus to glioma cell lines (A172 and U87) and glioma stem cells. ${ }^{33}$ Moreover, the authors investigated the effects that NDVinfected mesenchymal stem cells exhibit on cell death and self-renewal of glioma stem cells. They found that NDV (MTH-68/H strain) induced a dose-dependent cell death response in the cell lines as well as a low level of apoptosis and inhibition of glioma stem cell self-renewal. Their report demonstrated that glioma stem cells exhibit a reduced ability for self-renewal when infected with low titers of the virus. Moreover, their data showed that the virus exhibited no significant cytotoxic effect in human neural stem cells. ${ }^{33}$

\section{Combined Approaches}

Several investigators have evaluated NDV along with an additional adjuvant therapy in the treatment for GBM. Bai et al. demonstrated that combined therapy with temozolomide and NDV (LaSota strain) is more effective than either treatment alone for inducing apoptosis in human GBM cell lines (T98G, LN18, U87, U251). ${ }^{34}$ The virus inhibited Akt while activating AMPK when administered with temozolomide. Furthermore, the efficacy of this combined approach was confirmed in vivo with treatment groups demonstrating significantly smaller tumor volumes as well as significantly longer median survival times in tumor-bearing rats. Autopsy and blood analyses of healthy rats infected with the LaSota strain were unrevealing, indicating that this strain has minimal or no adverse effects. ${ }^{34}$ Alkassar et al. analyzed whether treatment with reovirus along with the NDV Hitchner B1 variant exhibits a synergistic oncolytic effect on GBM cell lines (U87 and U373) in culture and in a xenograft mouse model. ${ }^{35}$ They demonstrated that this combination of viral infection significantly enhanced oncolysis of GBM cell lines in vitro. Interestingly, a near-complete coinfection of all cells was reported with an increase in cell death, suggesting a synergistic intracellular effect between these viruses. Although the precise mechanisms of synergistic cooperation between viruses remained unclear, the authors hypothesized that it may be attributable to enhancement of the apoptotic pathway or utilization of the IFN pathway. ${ }^{35} \mathrm{Al}$-Shammari et al. investigated whether combining the Iraqi oncolytic attenuated AMHA1 strain (Iraq/Najaf/ICCMGR/2013 strain) enhances the efficiency of oncolysis in the human AMGM5 GBM cell line. ${ }^{36}$ They reported that combination therapy significantly reduced cancer cell viability with no cytotoxicity observed in normal cells. Moreover, in vivo data showed that combination therapy exhibited a higher growth inhibitory effect on transplanted tumors in mice than that with monotherapy alone. ${ }^{36}$

\section{Clinical Data}

The efficacy of NDV virotherapy has been studied in numerous clinical trials; however, intracranial tumors have often been excluded from these protocols. ${ }^{37-40} \mathrm{Al}-$ though the literature is scarce, there have been a few clinical studies that provide preliminary evidence of the oncolytic effects of NDV in GBM (Table 1).

\section{HUJ Strain}

Freeman et al. published results of a single-center, openlabel phase I/II clinical trial conducted in patients with recurrent GBM. ${ }^{41}$ This trial represented the first study to systemically administer NDV therapy in patients with recurrent GBM. The authors employed the NDV-HUJ strain, 


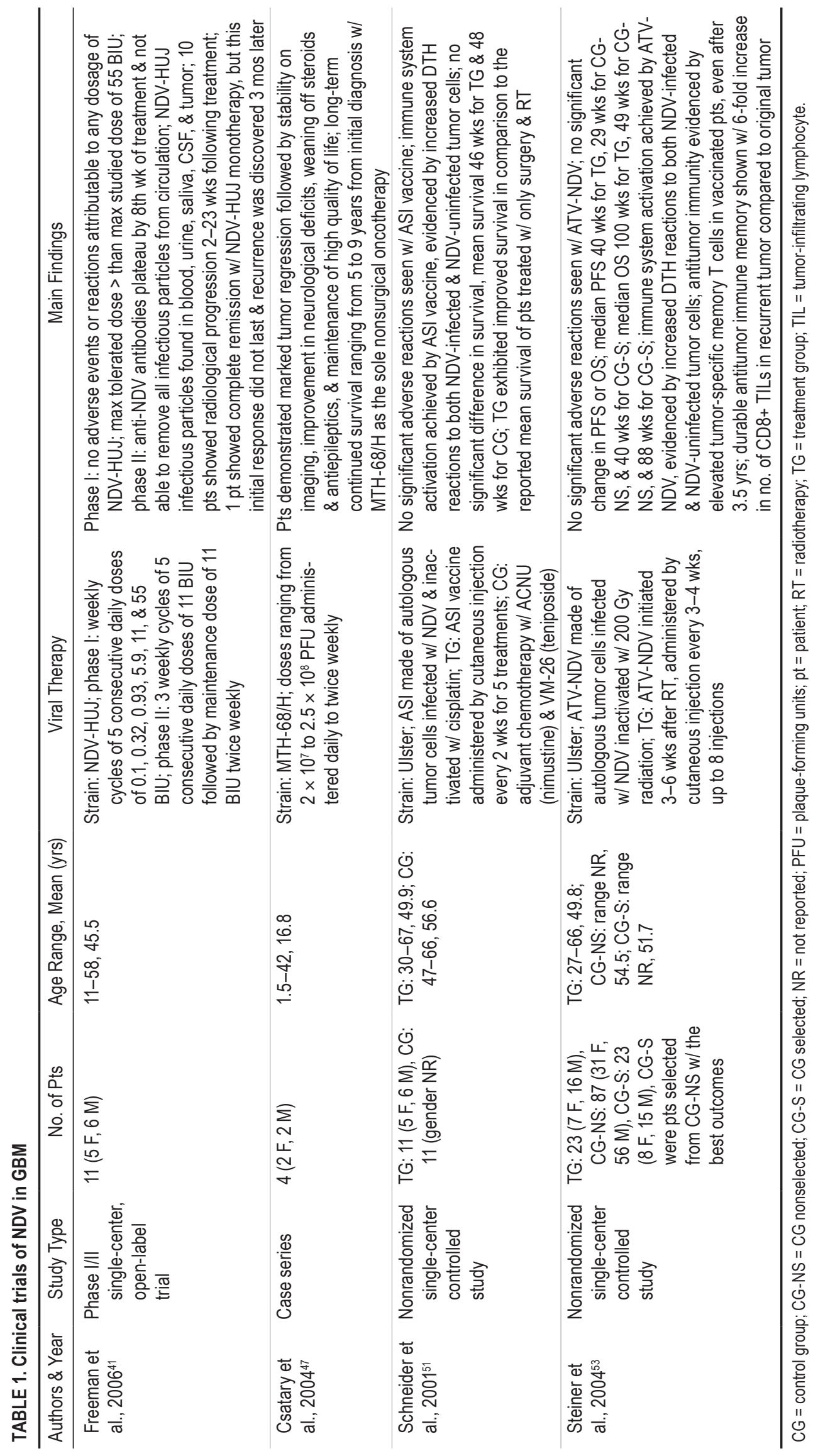



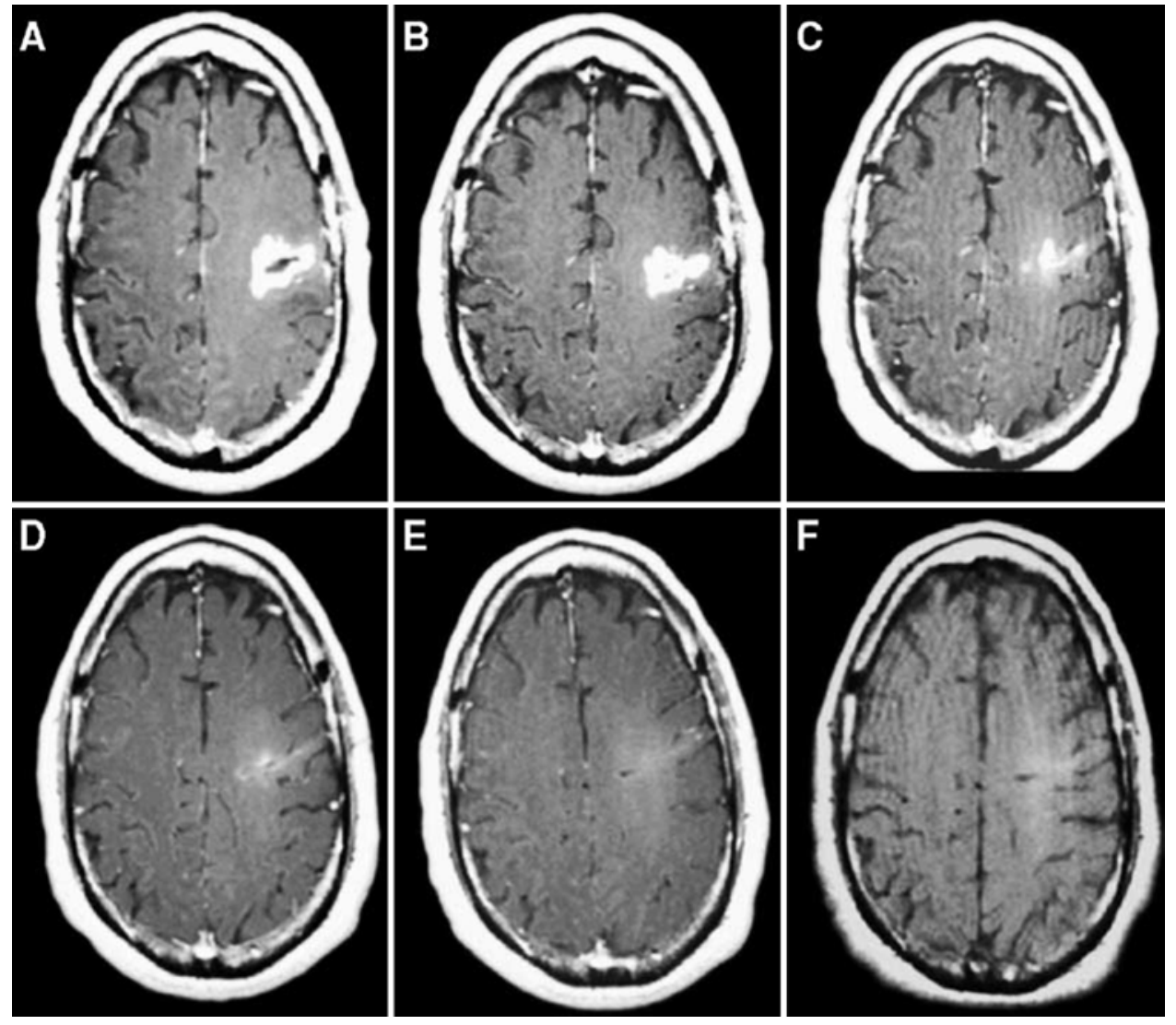

FIG. 2. Complete tumor response following NDV-HUJ treatment in case 9. A: MRI at baseline. B: Stable disease at the first followup. C: Partial response at the second follow-up. D: Partial response at 20 weeks from the start of virotherapy. E and F: Complete response at 25 and 30 weeks, respectively, from the start of virotherapy. Copyright American Society for Gene and Cell Therapy. Reproduced with permission from Freeman et al., 2006.41

a lentogenic strain developed at The Hebrew University of Jerusalem with known cytopathogenic effects observed in human cancer cell lines, as demonstrated with in vitro and in vivo studies. ${ }^{41-44}$ Eleven patients were enrolled in the study, including nine with de novo and two with secondary GBM. The authors investigated a constant dosing step consisting of 3 cycles of 11 BIU (billion infectious units), followed by a maintenance dose of 11 BIU twice weekly until there was radiographic evidence of tumor progression. Baseline anti-NDV antibodies were negative, yet antibody titers developed in all patients by the 5 th week of treatment and plateaued or decreased by the 8th week and remained low throughout repetitive dosing. Infectious NDV particles were found in blood, urine, and saliva, even after the development of antibodies, and were typically present for 9 days after the last systemic dose. This would suggest that anti-NDV antibodies are not able to remove all infectious particles from circulation. One patient demonstrated a significant tumor response during the first few dose cycles and complete tumor remission on maintenance dosing with a corresponding rapid neurological improvement and eventual independence from corticosteroids (Fig. 2). Unfortunately, the response was not enduring, and tumor recurrence was noted 3 months later. This finding is of particular importance, as complete remission of recurrent GBM is known to be rare even with current standard treatments. ${ }^{45,46}$ As the patient was not receiving any other therapies, the complete response can be reasonably attributed to the NDV-HUJ treatment. However, this response was unique, with the other study patients showing radiographic progression anywhere from 2 to 23 weeks following initiation of the NDV-based oncolytic therapy. ${ }^{41}$

\section{MTH-68/H Strain}

Csatary et al. used the MTH-68/H strain, first isolated in $1968,{ }^{8}$ to treat four patients with high-grade gliomas. ${ }^{47}$ This mesogenic strain of NDV has been shown to have potential immunostimulatory effects and direct cytotoxic effects on a variety of neoplastic cell types..$^{48}$ Fourteen patients with high-grade gliomas that had failed to respond to standard treatments were enrolled in the study. Only four patients were included in the final report since five patients had not responded to the treatment and had succumbed to their disease. ${ }^{47}$ One patient (case 1) was a 12-year-old male with a large left frontotemporal GBM that had progressed after surgical debulking, radiation, and multiple chemotherapy regimens. Over the course of 2 years, his tumor showed a significant response with approximately $95 \%$ 

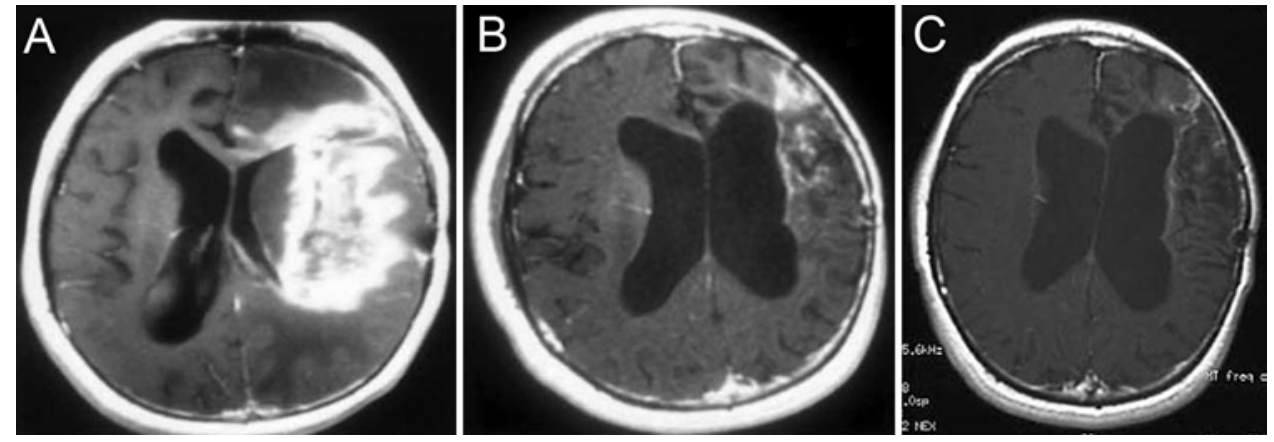

FIG. 3. Tumor response following MTH-68/H treatment. T1 postcontrast MRI scans from case 1. A: Scan from November 1996, 6 months after starting MTH-68/H treatment. B: Scan from September 1998, after MTH-68/H treatment showing definite shrinkage of the tumor and reduction of brain edema. C: Scan from September 2002 showing no change compared to panel B. Reprinted with permission from Springer Nature Customer Service Centre GmbH: Springer, Journal of Neuro-Oncology, MTH-68/H oncolytic viral treatment in human high-grade gliomas, LK Csatary, G Gosztonyi, J Szeberenyi, et al., @2004. https://www.springer.com/ journal/11060.

resolution, with a corresponding improvement in his neurological function, seizure control, and steroid dependence (Fig. 3). At the time of publication, Csatary et al. reported continued survival of all four patients ranging from 5 to 9 years from initial diagnosis. ${ }^{47}$ While these survival times would far exceed expectation in an adult population, three of the patients were children, a group known to have better prognoses, with a reported median survival of 13-73 months in pediatric GBM. ${ }^{49,50}$ However, the marked tumor regression, followed by stability on imaging, improvement in neurological deficits, the ability to wean off anticonvulsants and high-dose corticosteroids, and maintenance of a high quality of life in these patients with MTH-68/H as the sole nonsurgical oncotherapy, is quite remarkable. ${ }^{47}$

\section{Ulster Strain}

Schneider et al. published the first clinical study investigating the effects of active specific immunization (ASI) in GBM patients in 2001.51 The authors developed a patientderived vaccine composed of autologous irradiated tumor cells infected with NDV in order to harness the additive benefit of NDV's innate cytopathogenic and immunostimulatory effects along with presenting the diverse antigens specific to the tumor and effectively induce a potent and enduring immune response. ${ }^{51}$ The study group consisted of 11 consecutive patients with GBM (mean age 50 years) who underwent resection followed by adjuvant radiotherapy and ASI vaccination. This group was compared to 11 patients who were matched in age and neurological and functional status but who underwent adjuvant chemotherapy without virotherapy. The tumor vaccine was created from each patient's excised tumor, which was propagated in cell culture, infected with the NDV Ulster strain, and then inactivated through incubation with cytotoxic cisplatin. Type IV or delayed type hypersensitivity (DTH) reactions were measured at the injection sites and found to be prominent initially to NDV-infected tumor cells and continued to increase throughout the vaccination cycle. Importantly, while the initial DTH reaction to noninfected tumor cells was minimal, after the immunization cycle, the reaction became marked. The measurable increase in DTH reactions to NDV-infected and -uninfected tumor cells indicated that the immunization was effective in activating a peripheral immune response to GBM cells through T cell-mediated immunity. ${ }^{51,52}$ However, survival between the two groups did not vary significantly, with median and mean survival times of 46 and 60 weeks in the ASI group and 48 and 46.7 weeks in the group that received chemotherapy, respectively. The ASI group did exhibit better survival times than the reported mean survival of 38 weeks in patients treated exclusively with surgery and radiation. Therefore, the addition of ASI to resection and adjuvant radiation was concluded to be superior to surgery and radiation alone and comparable to the addition of adjuvant chemotherapy without the deleterious side effects. $^{51}$

A similar study using an NDV-infected antitumor vaccination (ATV-NDV) was published by Steiner et al. in $2004 .{ }^{53}$ They enrolled 23 patients with newly diagnosed GBM who underwent maximal resection, and they administered the ATV-NDV after completion of adjuvant radiation with cessation of steroids. The test group was compared to a nonselected control group of 87 patients who underwent maximal resection and radiation therapy alone. ${ }^{53}$ The ATV-NDV was created using fresh tumor samples that were grown in culture, incubated with the Ulster strain of NDV, and inactivated with ionizing radiation (200 Gy). Of note, chemotherapy was offered to patients only once tumor progression was identified. ${ }^{53,54}$ The median PFS was 40 weeks and the OS was 100 weeks for the vaccinated group, which were significantly longer than those for the nonselected control group. However, selection bias needed to be accounted for since complete resection was attained in $74 \%$ (17/23 patients) of test subjects and only $56 \%$ (49/87 patients) of control subjects. Therefore, the 23 control subjects with the best outcomes were selected as a new control group and found to have similar PFS of 40 weeks and OS of 88 weeks. ${ }^{53}$ While impact on survival was negligible, the authors were able to demonstrate activation of the systemic immune system as well as enduring antitumor immune memory. ${ }^{53}$ The measured DTH reactions increased significantly over time to both 

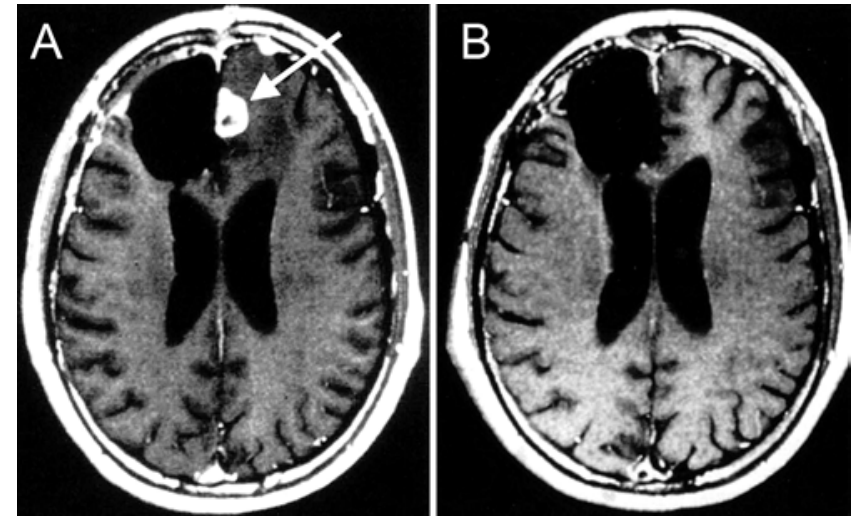

FIG. 4. Tumor regression following NDV-infected antitumor vaccine. A: MRI scan of the brain (T1 with gadolinium) of the patient in case 4 after standard treatment (surgery and radiotherapy). B: Six months after additional vaccination therapy. A tumor (arrow) that developed during radiotherapy completely disappeared after vaccination. Steiner $\mathrm{HH}$, Bonsanto MM, Beckhove P, et al., Antitumor vaccination of patients with glioblastoma multiforme: a pilot study to assess feasibility, safety, and clinical benefit, J Clin Oncol, 22, 21, pp 4272-4281. Reprinted with permission. @2004 American Society of Clinical Oncology. All rights reserved.

ATV-NDV injections and unmodified tumor cells, indicating systemic immune activation to GBM cells, similar to the findings from Schneider and colleagues..$^{51}$ In a subset of patients, the level of tumor-specific memory T cells, an indicator of antitumor immunity, was found to be elevated in vaccinated compared to nonvaccinated patients. ${ }^{55}$ In several patients who underwent reresections, the numbers of CD8+ tumor-infiltrating lymphocytes were compared between the original and recurrent tumors and found to remain low in nonvaccinated patients yet increased sixfold in vaccinated patients, which is evidence of durable antitumor immune memory (Fig. 4). ${ }^{53,55}$

\section{Conclusions}

NDV can selectively affect neoplastic cells, where it replicates without harming normal cells. The unique anticancer properties render NDV an excellent tool for immune-based therapies. While the use of NDV virotherapy in the treatment of GBM has not been extensively investigated, the few studies available have shown definite potential. Further investigation of NDV-based immunotherapy would ideally determine the optimum delivery and dosage for maximal efficacy and aim to delineate whether there are certain genetic or molecular tumor profiles that are more susceptible than others in the spirit of personalized therapy. When using isolated NDV strains for their cytotoxic and immunostimulatory effects, it would be important to determine an optimal dosing regimen that exploits the window between initial administration and the development of anti-NDV antibodies, thereby enhancing delivery of infectious particles to the tumor. With NDV-infected tumor vaccines, current data show that the peripheral immune response is strongly activated. It is important to also understand how robust the immune response is peripherally as well as within the tumor environment and what impact this activated immune system has on residual tumor cells and possible prevention of tumor recurrence. Furthermore, GBM cells are known to release various factors that suppress the immune response; therefore, targeting or eliminating these factors may be imperative to the efficacy of any immunomodulatory treatment. ${ }^{48}$ Clearly, additional work is needed to determine the role of the oncolytic NDV as an effective immunotherapeutic strategy against GBM.

Currently, there are no active clinical trials examining NDV as an oncolytic agent in the treatment of GBM. However, basic science research continues to thrive on this topic with several recent studies demonstrating promising results. With the advent of immunovirotherapy as a potent therapeutic strategy, further clinical studies will need to investigate the safety and efficacy of NDV as a treatment option for GBM.

\section{Acknowledgments}

Funding for this work was received from Carilion Clinic and Fralin Biomedical Research Institute at Virginia Tech Carilion School of Medicine.

\section{References}

1. Majewska P, Ioannidis S, Raza MH, et al. Postprogression survival in patients with glioblastoma treated with concurrent chemoradiotherapy: a routine care cohort study. CNS Oncol. 2017;6(4):307-313.

2. Cuoco JA, Benko MJ, Busch CM, et al. Vaccine-based immunotherapeutics for the treatment of glioblastoma: advances, challenges, and future perspectives. World Neurosurg. 2018;120:302-315.

3. Parajuli P, Anand R, Mandalaparty C, et al. Preferential expression of functional IL-17R in glioma stem cells: potential role in self-renewal. Oncotarget. 2016;7(5):6121-6135.

4. Chiocca EA, Rabkin SD. Oncolytic viruses and their application to cancer immunotherapy. Cancer Immunol Res. 2014; 2(4):295-300

5. Elankumaran S, Chavan V, Qiao D, et al. Type I interferonsensitive recombinant Newcastle disease virus for oncolytic virotherapy. J Virol. 2010;84(8):3835-3844.

6. Kaur B, Chiocca EA, Cripe TP. Oncolytic HSV-1 virotherapy: clinical experience and opportunities for progress. Curr Pharm Biotechnol. 2012;13(9):1842-1851.

7. Lun X, Chan J, Zhou H, et al. Efficacy and safety/toxicity study of recombinant vaccinia virus JX-594 in two immunocompetent animal models of glioma. Mol Ther. 2010;18(11): 1927-1936.

8. Csatary LK. Viruses in the treatment of cancer. Lancet. 1971; 2(7728):825.

9. Cassel WA, Garrett RE. Newcastle disease virus as an antineoplastic agent. Cancer. 1965;18:863-868.

10. Nandi S, Lesniak MS. Adenoviral virotherapy for malignant brain tumors. Expert Opin Biol Ther. 2009;9(6):737-747.

11. Sinkovics JG, Horvath JC. Newcastle disease virus (NDV): brief history of its oncolytic strains. J Clin Virol. 2000;16(1): $1-15$.

12. Zamarin D, Palese P. Oncolytic Newcastle disease virus for cancer therapy: old challenges and new directions. Future Microbiol. 2012;7(3):347-367.

13. Vigil A, Park MS, Martinez O, et al. Use of reverse genetics to enhance the oncolytic properties of Newcastle disease virus. Cancer Res. 2007;67(17):8285-8292.

14. Macpherson LW. Some observations on the epizootiology of NewCastle disease. Can J Comp Med Vet Sci. 1956;20(5): $155-168$ 
15. Abdullah JM, Mustafa Z, Ideris A. Newcastle disease virus interaction in targeted therapy against proliferation and invasion pathways of glioblastoma multiforme. BioMed Res Int. 2014;2014:386470.

16. Jin JH, Cheng JL, He ZR, et al. Different origins of Newcastle disease virus hemagglutinin-neuraminidase protein modulate the replication efficiency and pathogenicity of the virus. Front Microbiol. 2017;8:1607.

17. Reichard KW, Lorence RM, Cascino CJ, et al. Newcastle disease virus selectively kills human tumor cells. J Surg Res. 1992;52(5):448-453.

18. Krishnamurthy S, Takimoto T, Scroggs RA, Portner A. Differentially regulated interferon response determines the outcome of Newcastle disease virus infection in normal and tumor cell lines. $J$ Virol. 2006;80(11):5145-5155.

19. Washburn B, Schirrmacher V. Human tumor cell infection by Newcastle disease virus leads to upregulation of HLA and cell adhesion molecules and to induction of interferons, chemokines and finally apoptosis. Int J Oncol. 2002;21(1):85-93.

20. Elankumaran S, Rockemann D, Samal SK. Newcastle disease virus exerts oncolysis by both intrinsic and extrinsic caspasedependent pathways of cell death. J Virol. 2006;80(15): $7522-7534$

21. Fábián Z, Csatary CM, Szeberényi J, Csatary LK. p53-independent endoplasmic reticulum stress-mediated cytotoxicity of a Newcastle disease virus strain in tumor cell lines. $J \mathrm{Vi}$ rol. 2007;81(6):2817-2830.

22. Nelson CB, Pomeroy BS, Schrall K, et al. An outbreak of conjunctivitis due to Newcastle disease virus (NDV) occurring in poultry workers. Am J Public Health Nations Health. 1952;42(6):672-678.

23. Brown VR, Bevins SN. A review of virulent Newcastle disease viruses in the United States and the role of wild birds in viral persistence and spread. Vet Res (Faisalabad). 2017;48(1):68.

24. Matveeva OV, Guo ZS, Senin VM, et al. Oncolysis by paramyxoviruses: preclinical and clinical studies. Mol Ther Oncolytics. $2015 ; 2: 2$

25. Schirrmacher V. Fifty years of clinical application of Newcastle disease virus: time to celebrate! Biomedicines. 2016;4(3):E16.

26. Ali- Saeed R, Alabsi AM, Ideris A, et al. Evaluation of ultramicroscopic changes and proliferation of apoptotic glioblastoma multiforme cells induced by velogenic strain of Newcastle disease virus AF2240. Asian Pac J Cancer Prev. 2019; 20(3):757-765.

27. Yuan P, Paterson RG, Leser GP, et al. Structure of the ulster strain Newcastle disease virus hemagglutinin-neuraminidase reveals auto-inhibitory interactions associated with low virulence. PLoS Pathog. 2012;8(8):e1002855.

28. Imre G. Cell death signalling in virus infection. Cell Signal. 2020;76:109772.

29. Koks CA, Garg AD, Ehrhardt M, et al. Newcastle disease virotherapy induces long-term survival and tumor-specific immune memory in orthotopic glioma through the induction of immunogenic cell death. Int J Cancer. 2015;136(5):E313-E325.

30. Zamarin D, Martínez-Sobrido L, Kelly K, et al. Enhancement of oncolytic properties of recombinant Newcastle disease virus through antagonism of cellular innate immune responses. Mol Ther. 2009;17(4):697-706.

31. García-Romero N, Palacín-Aliana I, Esteban-Rubio S, et al. Newcastle disease virus (NDV) oncolytic activity in human glioma tumors is dependent on CDKN2A-Type I IFN gene cluster codeletion. Cells. 2020;9(6):E1405.

32. Xie Q, Mittal S, Berens ME. Targeting adaptive glioblastoma: an overview of proliferation and invasion. Neuro Oncol. 2014;16(12):1575-1584.

33. Kazimirsky G, Jiang W, Slavin S, et al. Mesenchymal stem cells enhance the oncolytic effect of Newcastle disease virus in glioma cells and glioma stem cells via the secretion of TRAIL. Stem Cell Res Ther. 2016;7(1):149.
34. Bai Y, Chen Y, Hong X, et al. Newcastle disease virus enhances the growth-inhibiting and proapoptotic effects of temozolomide on glioblastoma cells in vitro and in vivo. Sci Rep. 2018;8(1):11470.

35. Alkassar M, Gärtner B, Roemer K, et al. The combined effects of oncolytic reovirus plus Newcastle disease virus and reovirus plus parvovirus on U87 and U373 cells in vitro and in vivo. J Neurooncol. 2011;104(3):715-727.

36. Al-Shammari AM, Jalill RDA, Hussein MF. Combined therapy of oncolytic Newcastle disease virus and rhizomes extract of Rheum ribes enhances cancer virotherapy in vitro and in vivo. Mol Biol Rep. 2020;47(3):1691-1702.

37. Lorence RM, Katubig BB, Reichard KW, et al. Complete regression of human fibrosarcoma xenografts after local Newcastle disease virus therapy. Cancer Res. 1994;54(23): 6017-6021.

38. Pecora AL, Rizvi N, Cohen GI, et al. Phase I trial of intravenous administration of PV701, an oncolytic virus, in patients with advanced solid cancers. J Clin Oncol. 2002;20(9): 2251-2266.

39. Phuangsab A, Lorence RM, Reichard KW, et al. Newcastle disease virus therapy of human tumor xenografts: antitumor effects of local or systemic administration. Cancer Lett. 2001;172(1):27-36.

40. Zhao L, Liu H. Newcastle disease virus: a promising agent for tumour immunotherapy. Clin Exp Pharmacol Physiol. 2012;39(8):725-730.

41. Freeman AI, Zakay-Rones Z, Gomori JM, et al. Phase I/II trial of intravenous NDV-HUJ oncolytic virus in recurrent glioblastoma multiforme. Mol Ther. 2006;13(1):221-228.

42. Yurchenko KS, Zhou P, Kovner AV, et al. Oncolytic effect of wild-type Newcastle disease virus isolates in cancer cell lines in vitro and in vivo on xenograft model. PLoS One. 2018; 13(4):e0195425.

43. Yaacov B, Eliahoo E, Lazar I, et al. Selective oncolytic effect of an attenuated Newcastle disease virus (NDV-HUJ) in lung tumors. Cancer Gene Ther. 2008;15(12):795-807.

44. Lazar I, Yaacov B, Shiloach T, et al. The oncolytic activity of Newcastle disease virus NDV-HUJ on chemoresistant primary melanoma cells is dependent on the proapoptotic activity of the inhibitor of apoptosis protein Livin. J Virol. 2010; 84(1):639-646.

45. Wong ET, Hess KR, Gleason MJ, et al. Outcomes and prognostic factors in recurrent glioma patients enrolled onto phase II clinical trials. J Clin Oncol. 1999;17(8):2572-2578.

46. Costa E, Lawson TM, Lelotte J, et al. Long-term survival after glioblastoma resection: hope despite poor prognosis factors. J Neurosurg Sci. 2019;63(3):251-257.

47. Csatary LK, Gosztonyi G, Szeberenyi J, et al. MTH-68/H oncolytic viral treatment in human high-grade gliomas. $J$ Neurooncol. 2004;67(1-2):83-93.

48. Csatary LK, Moss RW, Beuth J, et al. Beneficial treatment of patients with advanced cancer using a Newcastle disease virus vaccine (MTH-68/H). Anticancer Res. 1999;19(1B): 635-638.

49. Perkins SM, Rubin JB, Leonard JR, et al. Glioblastoma in children: a single-institution experience. Int J Radiat Oncol Biol Phys. 2011;80(4):1117-1121.

50. Das KK, Mehrotra A, Nair AP, et al. Pediatric glioblastoma: clinico-radiological profile and factors affecting the outcome. Childs Nerv Syst. 2012;28(12):2055-2062.

51. Schneider T, Gerhards R, Kirches E, Firsching R. Preliminary results of active specific immunization with modified tumor cell vaccine in glioblastoma multiforme. J Neurooncol. 2001;53(1):39-46.

52. Kniker WT, Anderson CT, Roumiantzeff M. The multi-test system: a standardized approach to evaluation of delayed hypersensitivity and cell-mediated immunity. Ann Allergy. 1979;43(2):73-79. 
53. Steiner HH, Bonsanto MM, Beckhove P, et al. Antitumor vaccination of patients with glioblastoma multiforme: a pilot study to assess feasibility, safety, and clinical benefit. J Clin Oncol. 2004;22(21):4272-4281.

54. Stupp R, Mason WP, van den Bent MJ, et al. Radiotherapy plus concomitant and adjuvant temozolomide for glioblastoma. N Engl J Med. 2005;352(10):987-996.

55. Feuerer M, Beckhove P, Bai L, et al. Therapy of human tumors in NOD/SCID mice with patient-derived reactivated memory T cells from bone marrow. Nat Med. 2001;7(4): $452-458$.

\section{Disclosures}

The authors report no conflict of interest concerning the materials or methods used in this study or the findings specified in this paper.

\section{Author Contributions}

Conception and design: Mittal. Acquisition of data: Cuoco, Rogers. Analysis and interpretation of data: all authors. Drafting the article: Cuoco, Rogers. Critically revising the article: all authors. Reviewed submitted version of manuscript: all authors. Approved the final version of the manuscript on behalf of all authors: Mittal. Administrative/technical/material support: Mittal. Study supervision: Mittal.

\section{Correspondence}

Sandeep Mittal: Virginia Tech Carilion School of Medicine, Roanoke, VA. sandeepmittal@vt.edu. 